\title{
Vida en anaquel de galletas saladas utilizando pruebas aceleradas
}

\author{
Shelf life of crackers using accelerated tests \\ Gustavo Gavino Puma Isuiza ${ }^{1 *}$; Jhoselyn Floryan Liñan Perez ${ }^{1}$; Ibeth Coavoy Sánchez ${ }^{1}$; Jorge Coronado Olano; \\ Walter Francisco Salas Valerio ${ }^{2}$, Luis Fernando Vargas Delgado ${ }^{2}$ \\ * Autor de correspondencia
}

\begin{abstract}
Resumen
El presente trabajo de investigación tuvo como objetivo estimar el tiempo de vida en anaquel de galletas de soda a través del empleo de pruebas aceleradas. Las galletas se sometieron a ambientes de almacenamiento de 35 , 45 y 55 ${ }^{\circ} \mathrm{C}$ a 80 por ciento de humedad relativa en las tres cámaras. Se evaluaron los parámetros: humedad, actividad de agua y dureza instrumental, los cuales fueron modelados a una reacción de orden uno reportando que el tiempo de vida útil de las galletas saladas, para cada parámetro, fue de 123, 179 y 271 días, respectivamente. El tiempo de vida útil estaba predispuesto por la humedad crítica $(6,38 \%$ b.h.), debido a que fue el parámetro que se vio más afectado por las condiciones de almacenamiento. Finalmente, se realizó un recuento de mohos, para evaluar la inocuidad del alimento, encontrándose que el alimento se mantuvo dentro de los límites establecidos por la NTP con valores $<10$ al inicio y al final del almacenamiento.
\end{abstract}

Palabras clave: Parámetro crítico; Arrhenius; Energía de activación; orden de reacción; vida en anaquel.

\begin{abstract}
The objective of this research was to estimate the shelf life of soda crackers through the use of accelerated tests. The cookies were subjected to storage environments of 35,45 and $55^{\circ} \mathrm{C}$ at 80 percent relative humidity in the three chambers. The parameters were evaluated: humidity, water activity and instrumental hardness, which were modeled to a reaction of order one reporting that the shelf life of the salty cookies, for each parameter, was 123, 179 and 271 days, respectively . The useful life time was predisposed by the critical humidity $(6.38 \%$ b.h. $)$, because it was the parameter that was most affected by the storage conditions. Finally, a count of molds was carried out to evaluate the safety of the food, finding that the food remained within the limits established by the NTP with values $<10$ at the beginning and at the end of storage.
\end{abstract}

Keywords: Critical parameter; Arrhenius; activation energy; reaction order; shelf life.

\section{Introducción}

Las galletas, son un vehículo de nutrientes (Granito et al., 2006), están hechas a base de harina, grasa y sal; opcionalmente, se puede añadir una mínima cantidad de azúcar. En cien gramos, las galletas de tipo soda, contienen $433 \mathrm{Kcal}$ de energía, $4.8 \mathrm{~g}$ de agua, $68 \mathrm{~g}$ de carbohidratos totales y pequeñas cantidades de micronutrientes y macronutrientes fundamentales para la alimentación del ser humano como calcio, fósforo, zinc, hierro, retinol, tiamina, riboflbina y niacina (Gimferrer, 2007 citado por García, 2016). La galleta "Soda Field" fue una de las marcas con mayor presencia en el mercado peruano, con un $27,3 \%$ de preferencia, según CPI (2012). Por su alto consumo y preferencia, es necesario el estudio de la vida útil de las galletas saladas, las mismas que dependen de una serie de factores como la materia prima, la formulación del producto, el proceso aplicado, las condiciones sanitarias del proceso, el envasado, el almacenamiento, la distribución y las prácticas de los consumidores (Carrillo y Reyes, 2013), que permite al producto conservar sus características sensoriales (Hough, 2010). Por ello, la industria de alimentos tiene la necesidad de conocer, en un corto periodo de tiempo, la información necesaria para determinar la vida útil de sus productos (Qi et al., 2014).

Existen tres métodos de evaluación para determinar la vida útil de un producto. Las pruebas de vida útil a tiempo real, los datos que brindan son buenos, pero en algunas ocasiones se requiere de un tiempo prolongado para la obtención del resultado final (Udomkun et al., 2016). La simulación y la estimación basada en modelos, es una técnica que implica la combinación de expresiones de la sensibilidad del producto, la eficacia del empaque y la

\footnotetext{
${ }^{1}$ Maestrista de Tecnología de Alimentos, Escuela de Posgrado, Universidad Nacional Agraria La Molina, Apartado postal 12-056- La Molina, Lima, Perú. Email: 20090468@lamolina.edu.pe

2Departamento de Ingeniería, Facultad de Industrias Alimentarias, Universidad Nacional Agraria La Molina, Lima, Perú. Email: wfsalas@lamolina. edu.pe
} 
severidad del medio ambiente en un modelo matemático. Las pruebas aceleradas, permiten obtener datos en tiempos relativamente cortos, consisten en incubar el alimento bajo condiciones controladas y a diferentes temperaturas (Sánchez-González y Pérez, 2016). Estas temperaturas, deben ser mayores a las de almacenamiento y comercialización para que las reacciones de deterioro se aceleren y se obtenga el resultado en un periodo significativamente más cortos (Rodríguez, 2004) que el período de vida útil real del producto (Rao et al., 2012; Derossi et al., 2016).

El objetivo de este estudio fue determinar la vida útil en anaquel de galletas saladas mediante pruebas aceleradas; empleando el porcentaje de humedad, actividad de agua y dureza instrumental como indicadores críticos de deterioro.

\section{Materiales y métodos}

\section{Muestra}

Para el presente estudio se utilizaron galletas de soda marca "Soda Field", en presentación de 34 g, los cuales procedían de la empresa Mondelez Perú S.A. ubicado en la ciudad de Lima - Perú. Cada una de las muestras pertenecía a un mismo lote, con las mismas condiciones de proceso y materia prima.

\section{Diseño experimental}

Se aplicó la metodología de análisis de supervivencia con pruebas aceleradas. Se tomaron las mismas cantidades de muestras de galletas, procediendo a colocarlas en cámaras de climatización para el estudio de vida útil acelerado, a una humedad relativa del $80 \%$ y a 35,45 y $55{ }^{\circ} \mathrm{C}$., respectivamente, de donde se fueron retirando según el tiempo establecido para cada temperatura (Tabla 1).

Tabla 1. Esquema de toma de muestra y análisis, realizados a las muestras de galletas de soda, almacenadas a 35, 45 y $55^{\circ} \mathrm{C}$ a $80 \%$ de humedad relativa.

\begin{tabular}{|c|c|c|c|}
\hline Tratamientos & $\begin{array}{c}\mathrm{A} \\
55^{\circ} \mathrm{C}-80 \% \mathrm{HR}\end{array}$ & $\begin{array}{c}\mathrm{B} \\
45^{\circ} \mathrm{C}-80 \% \\
\mathrm{HR}\end{array}$ & $\begin{array}{c}\mathrm{C} \\
35{ }^{\circ} \mathrm{C}-80 \% \\
\mathrm{HR}\end{array}$ \\
\hline $\begin{array}{c}\text { Ensayo } \\
\text { Experimental }\end{array}$ & $\begin{array}{c}\text { Toma de } \\
\text { muestra: } 8, \\
\text { intercalando } 3 \\
\text { días }\end{array}$ & $\begin{array}{c}\text { Toma de } \\
\text { muestra: } 8, \\
\text { intercalando } 5 \\
\text { días }\end{array}$ & $\begin{array}{c}\text { Toma de } \\
\text { muestra: } 8 \text {, } \\
\text { intercalado } 7 \\
\text { días }\end{array}$ \\
\hline Se evaluó & $\begin{array}{c}\text { Humedad } \\
\text { Actividad de } \\
\text { Agua } \\
\text { Textura } \\
\text { instrumental } \\
\text { Recuento de } \mathrm{N} \\
\text { fina }\end{array}$ & $\begin{array}{l}\text { Humedad } \\
\text { Actividad de } \\
\text { Agua } \\
\text { Textura } \\
\text { instrumental }\end{array}$ & $\begin{array}{l}\text { Humedad } \\
\text { Actividad de } \\
\text { Agua } \\
\text { Textura } \\
\text { instrumental } \\
\text { Al inicio y al } \\
\text { to }\end{array}$ \\
\hline
\end{tabular}

\section{Análisis Sensorial}

Las muestras fueron acondicionadas a $80{ }^{\circ} \mathrm{C}$ durante 15 a 20 minutos en baño maría (Shaking Water Bath Order No 1083) con la finalidad de generar diferentes valores de los parámetros críticos. Luego, fueron almacenadas en bolsas de polietileno de baja densidad. Para la prueba sensorial se reclutó a 100 estudiantes de la Universidad Nacional Agraria La Molina, quienes eran consumidores habituales de galletas "Soda Field". Este número es superior a los considerados por Sánchez-González y Pérez (2016). La prueba de aceptación del producto en estudio, se realizó por decisión simple: "sí" o "no" consumirían el producto; evaluando por la misma cantidad de consumidores durante el tiempo que duraron las pruebas aceleradas; las muestras de galletas fueron codificadas con dígitos y brindadas al consumidor a temperatura ambiente (Hough et al., 2003). Se consideró el $75 \%$ de aceptación en la determinación de los parámetros críticos $\left(\mathrm{A}_{\mathrm{w}}\right.$, humedad $\mathrm{y}$ dureza instrumental).

\section{Determinación de la humedad}

Se determinó la humedad de las galletas de acuerdo a la Norma Técnica Peruana 206.011 (1981).

\section{Determinación de la actividad de agua}

En una cubeta de plástico especial del equipo de medida Aqua Lab Water Activity (Aqua Lab, Series tres TE) se depositó la muestra de galleta molida y se procedió a realizar la medición. Las mediciones se realizaron por triplicado, empleando un paquete de galletas para cada repetición.

\section{Determinación de la dureza instrumental}

Debido a que las galletas presentan una baja humedad, típicamente inferior al 5\%, y son de espesor delgado, les confiere las características de frescura y dureza (Manley, 2000; Subramaniam y Wareing, 2016). La dureza de los productos de panadería se puede evaluar usando algún tipo de prueba de punción. Esta prueba es frecuentemente usada en galletas saladas y es útil para seguir los cambios que surgen en la migración de la humedad (Cauvain y Young, 2009).

La dureza instrumental se realizó con el equipo Universal Testing Machine Instron ${ }^{\circledR}$ (Tabletop Model 3365), utilizando los siguientes accesorios: base CAT NO S5406A Max Carga $2 \mathrm{KN}$, punta de penetración de $4,31 \mathrm{~mm}$ de diámetro. Se corrió en el programa Instron Bluehill empleando los siguientes datos de programación: velocidad $20 \mathrm{~mm} / \mathrm{min}$, pre carga de 10 gramos fuerza (gf) compresión de $50 \%$ y la punta de penetración inicia en $19 \mathrm{dm}$. Se hallaron las dimensiones de la galleta (ancho, largo, espesor) con la ayuda de un vernier para cargarlas en el programa. El programa calculó la dureza en gramos fuerza como punto máximo de la curva. Se realizaron tres repeticiones de cada paquete de galletas y se usaron tres paquetes por cada temperatura de almacenamiento. 


\section{Recuento de mohos}

El recuento de mohos de las galletas se realizó de acuerdo al método de ensayo ISO 21527-2. AOAC 2014.05, FDA/ BAM, AACC 42-50.01, que son establecidas en la Norma Técnica Peruana 206.001 (2016).

\section{Determinación del tiempo de vida mediante pruebas aceleradas}

Se determinó la orden de reacción en función del $\mathrm{r}^{2}$ al relacionar el valor crítico (A) vs tiempo de almacenamiento (orden cero) y LnA vs tiempo de almacenamiento (orden uno). Se escogió el orden de reacción que correspondió al mayor $\mathrm{r}^{2}$.

$$
\frac{d A}{d t}= \pm k A^{n} \quad \text { Ec. } 1
$$

Ecuación de cinética de reacción; donde n: orden de la reacción (0 ó 1)

Ecuación de la cinética de reacción linealizada para orden uno:

$$
\ln A=\ln A_{0} \pm k t \quad \text { Ec. } 2
$$

Donde:

A: Atributo

$\mathrm{A}_{0}$ : Atributo inicial.

k: Constante de cinética de reacción.

t: Tiempo.

\section{Determinación de la Ea y $\mathbf{K}_{20}$}

Se determinó la energía de activación (Ea) de la gráfica Lnk vs. $1 / \mathrm{T}$ abs $\left({ }^{\circ} \mathrm{K}\right)$; donde $\mathrm{Ea} / \mathrm{R}$ fue la pendiente y $\mathrm{Lnk}_{0}$, el intercepto.

$$
L n k=L n k_{0}-\frac{E a}{R T}
$$

Donde:

$\mathrm{k}_{\mathrm{o}}$ : Factor pre-exponencial

Ea: Energía de activación $(\mathrm{KJ} / \mathrm{mol})$

$\mathrm{R}$ : Constante universal de los gases $(0.008314 \mathrm{KJ} / \mathrm{mol} \mathrm{K})$

$\mathrm{T}$ : Temperatura absoluta $(\mathrm{K})$

Luego se emplearon los valores hallados de Ea y $\mathrm{K}_{0}$, para calcular el $\mathrm{k}_{20}$ donde la temperatura absoluta fue $293{ }^{\circ} \mathrm{K}$; empleando la siguiente ecuación:

$$
k=k_{0} e^{-\frac{E a}{R T}} \quad \text { Ec. } 4
$$

Conociendo todos los parámetros se determinó el tiempo de vida útil de la galleta salada mediante la Ecuación 2. Los datos fueron procesados con el Software Sigma Plot 11.0 , versión prueba.

\section{Resultados y discusión}

\section{Parámetros Críticos}

La prueba sensorial con 100 consumidores determinaron los valores críticos para cada característica en estudio que fueron utilizados para determinar el tiempo de vida de las galletas de soda. Los valores críticos que se obtuvieron fueron: 6,3\% de humedad (b.h.) 0,474 de actividad de agua y un promedio de dureza instrumental de 471,23 gf.. El valor de la humedad crítica es similar a lo reportado por Sirpatrawan (2009) en galleta de soda $(6,8 \%)$, quien además menciona que hasta este valor de humedad las galletas son aceptadas por los consumidores, con un nivel más alto disminuye la aceptación debido al cambio de humedad que tiene consecuencia en la crocantes de la galleta.

\section{Determinación del tiempo de vida a partir del porcentaje de humedad (b.s)}

En la Figura 1 se presenta la variación de la humedad en base seca (b.s.) de las galletas de soda respecto al tiempo de almacenamiento, para las temperaturas de 35 , 45 y $55^{\circ} \mathrm{C}$, observando que a la temperatura de $55^{\circ} \mathrm{C}$ la humedad aumenta rápidamente en comparación de las otras dos temperaturas. Esto se debe a que el aumento de la temperatura acelera el fenómeno de transferencia de masa hacia el interior del empaque por lo que provoca una ganancia de agua en la galletas (Bustamante, 2015).

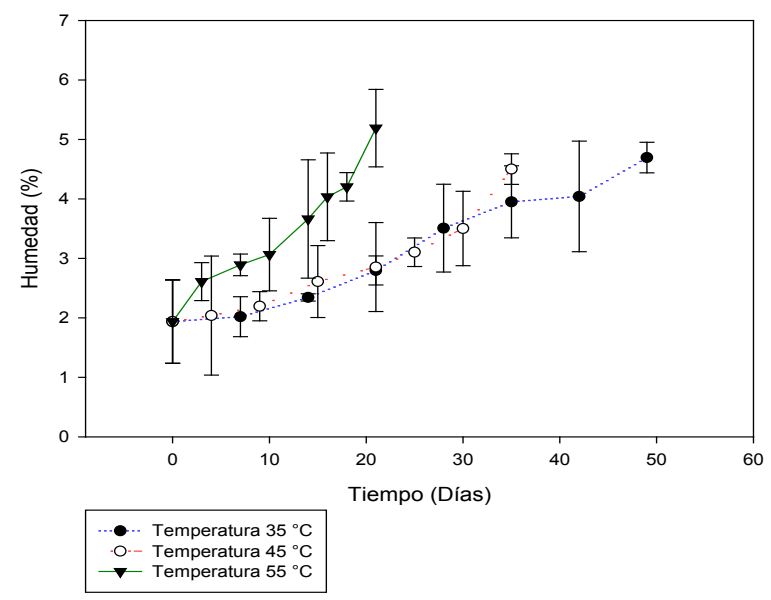

Figura 1. Tendencia de los valores del porcentaje de humedad (b. s.), respecto al tiempo de almacenamiento de las galletas a 35,45 y $55^{\circ} \mathrm{C}$.

Los valores del $\mathrm{r}^{2}$ establecieron que el orden de la reacción fue uno para las tres temperaturas. En la Figura 2 se muestra la regresión lineal usando el logaritmo neperiano de la humedad (b. s.) respecto al tiempo de almacenamiento a las tres temperaturas en estudio. 


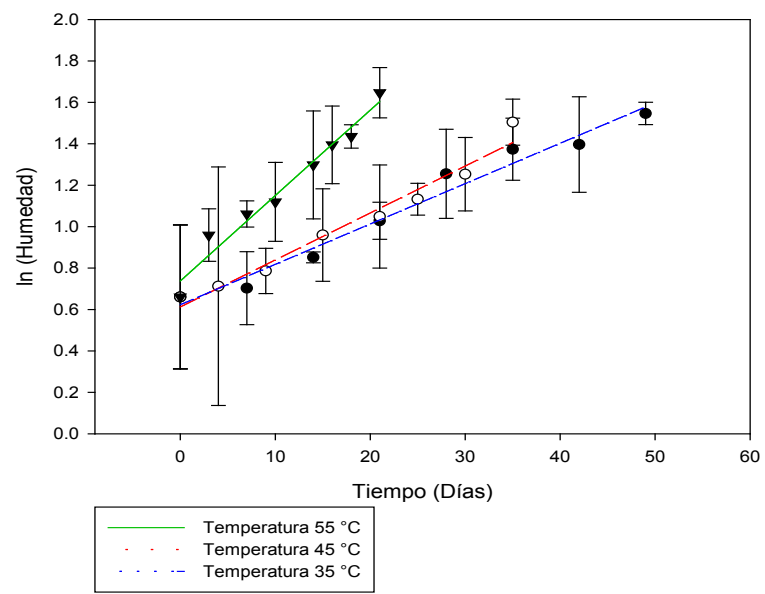

Figura 2. Relación entre $\operatorname{Ln}($ humedad) en función del tiempo de almacenamiento (días) de las galletas a 35, 45 y $55^{\circ} \mathrm{C}$

Hao et al. (2016), evaluaron la vida útil de galletas en empaques sensibles a la humedad determinando que el orden de reacción para la ganancia de agua a lo largo del tiempo fue de orden uno. Labuza (1979), indica que un efecto importante de la humedad sobre las velocidades de reacción podría ser el cambiar el orden de reacción. Dicho autor indica que, lamentablemente, la mayoría de los estudios bibliográficos sobre las reacciones alimenticias no permiten que la degradación avance suficientemente para discernir cuál es el verdadero orden.

Los valores de coeficiente de determinación, pendiente (k) e intercepto de cada temperatura (Tabla 2) se obtuvieron al modelar los datos a una ecuación linealizada de orden uno, donde la abscisa fue el tiempo y la ordenada el logaritmo neperiano del porcentaje de humedad en base seca.

Se utilizó la ecuación de Arrhenius para hallar la constante de velocidad $\mathrm{k}$ a $20^{\circ} \mathrm{C}$, y así determinar el tiempo de vida útil de la galleta de soda (Tabla 3), obteniendo un $\mathrm{k}$ de 0,0097 , resultado similar al reportado por BustamanteOyague (2015) de 0,009.

Bustamante-Oyague (2015) evaluó muestras de galleta de soda en envase de polipropileno a 30, 40 y $50{ }^{\circ} \mathrm{C}$ mediante el modelo de Arrhenius, reportando que la Energía de Activación obtenida fue de 24,41 KJ/mol. Dicho valor es similar a lo obtenido en el Tabla 3.

Según el registro sanitario "H6300210N-NAKAFO" (DIGESA, 2015), el tiempo de vida útil de las galletas de soda es de siete meses, lo cual difiere al resultado obtenido que es de cuatro meses, esto se debe a que la humedad crítica para galletas de soda en el Perú es del 12\% (INACAL, 2016), mucho mayor a la humedad crítica establecida por los consumidores $(6,38 \%)$. Por otro lado, Bustamante-Oyague (2015) trabajó con una humedad crítica comercial de $8,0 \%$.
Tabla 2. Valores de k para cada temperatura $(35,45$ y 55 ${ }^{\circ} \mathrm{C}$ )

\begin{tabular}{cccc}
\hline $\mathrm{T}\left({ }^{\circ} \mathrm{C}\right)$ & $\mathrm{r}^{2}$ & $\begin{array}{c}\text { Pendiente }(\mathrm{k}) \\
\left(\mathrm{dí}^{-1}\right)\end{array}$ & Intercepto \\
\hline 35 & 0,9729 & 0,0195 & 0,6246 \\
45 & 0,9685 & 0,0227 & 0,6133 \\
55 & 0,9679 & 0,0413 & 0,7383 \\
\hline
\end{tabular}

Tabla 3. Determinación de la energía de activación (Ea), $\mathrm{k}_{0}$, $\mathrm{k}_{20}\left(20^{\circ} \mathrm{C}\right)$ y el tiempo de vida con respecto al contenido de humedad de la galleta de soda.

\begin{tabular}{cccccc}
\hline -Ea (KJ/mol) & $\mathrm{K}_{0}$ & $\begin{array}{c}\mathrm{k}_{20} \\
(\mathrm{día}-1)\end{array}$ & $\begin{array}{c}\mathrm{H}_{\mathrm{c}} \\
(\%)\end{array}$ & $\begin{array}{c}\mathrm{t}_{20} \\
(\text { días }\end{array}$ & $\begin{array}{c}\mathrm{t}_{20} \\
(\mathrm{meses})\end{array}$ \\
\hline 31,2897 & 3660,8 & 0,0097 & 6,38 & 123 & 4 \\
\hline
\end{tabular}

$\mathrm{Hc}$, humedad critica; Ea, energía de activación; $\mathrm{k}_{20}$, constante de cinética de reacción a $20{ }^{\circ} \mathrm{C} ; \mathrm{K}_{0}$, Factor pre-exponencial; $\mathrm{t}_{20}$, tiempo de vida a 20 ${ }^{\circ} \mathrm{C}$

\section{Determinación del tiempo de vida a partir de la actividad de agua $\left(\mathrm{A}_{\mathrm{w}}\right)$}

En la Figura 3 se presenta la variación de la actividad de agua $\left(\mathrm{A}_{\mathrm{w}}\right)$ de las galletas de soda respecto al tiempo de almacenamiento a 35,45 y $55^{\circ} \mathrm{C}$. Se observa que a $55^{\circ} \mathrm{C}$ la $\mathrm{A}_{\mathrm{w}}$ del alimento aumentó en mayor grado a comparación de las temperaturas de 35 y $45^{\circ} \mathrm{C}$. Esta tendencia puede deberse a lo estudiado por Scott y Bernard (1983); citados por Espinoza (1993), quienes mencionan que la $A_{w}$ depende de la temperatura ya que la proporción de agua libre en el alimento varía con la temperatura. Asimismo indican que la influencia de la temperatura sobre la $\mathrm{A}_{\mathrm{w}}$ es mayor a temperaturas superiores a $30{ }^{\circ} \mathrm{C}$, siendo esta influencia mayor en alimentos de niveles bajos de $A_{w}$; mientras que en alimentos con altos niveles de Aw el efecto es mínimo.

Además se observa que los valores obtenidos no se ajustan a una línea recta, por lo cual se determinaron los valores de $\mathrm{r}^{2}$ (Tabla 4) que establecieron que el orden de la reacción fue uno para las tres temperaturas.

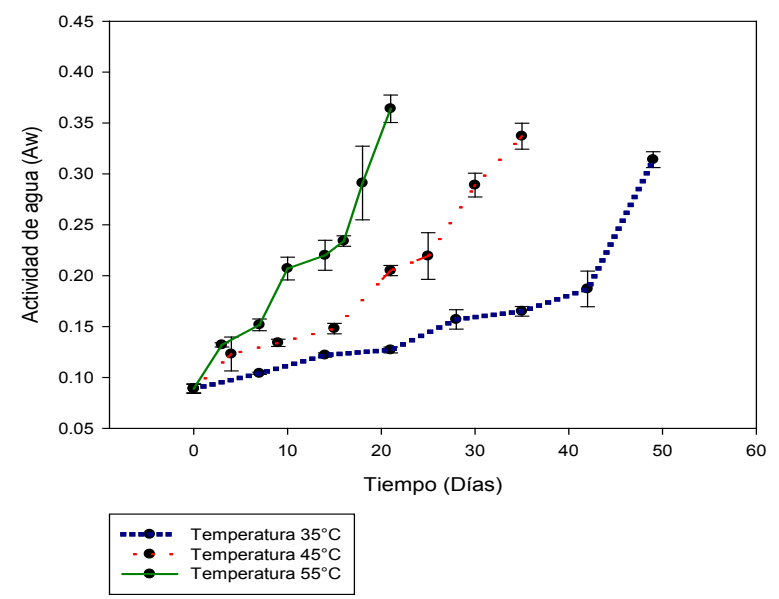

Figura 3. Tendencia de los valores de la actividad de agua respecto al tiempo de almacenamiento de las galletas a 35, 45 y $55^{\circ} \mathrm{C}$. 
Tabla 4. Valores de K para cada temperatura (35, 45 y 55 $\left.{ }^{\circ} \mathrm{C}\right)$

\begin{tabular}{cccc}
\hline $\mathrm{T}\left({ }^{\circ} \mathrm{C}\right)$ & $\mathrm{r}^{2}$ & $\begin{array}{c}\text { Pendiente } \\
\left(\text { día }^{-1}\right)\end{array}$ & Intercepto \\
\hline 35 & 0,9144 & 0,0219 & $-2,4538$ \\
45 & 0,9777 & 0,0358 & $-2,3548$ \\
55 & 0,9615 & 0,0598 & $-2,3049$ \\
\hline
\end{tabular}

Los valores de $\mathrm{r}^{2}$, pendiente $(\mathrm{k})$ e intercepto de cada temperatura se obtuvieron al modelar los datos a una ecuación linealizada de orden de reacción uno, donde la abscisa fue el tiempo y la ordenada el logaritmo neperiano de la actividad de agua.

En la Figura 4 se muestra la regresión lineal usando el logaritmo neperiano de la actividad de agua respecto al tiempo de almacenamiento a las tres temperaturas en estudio.

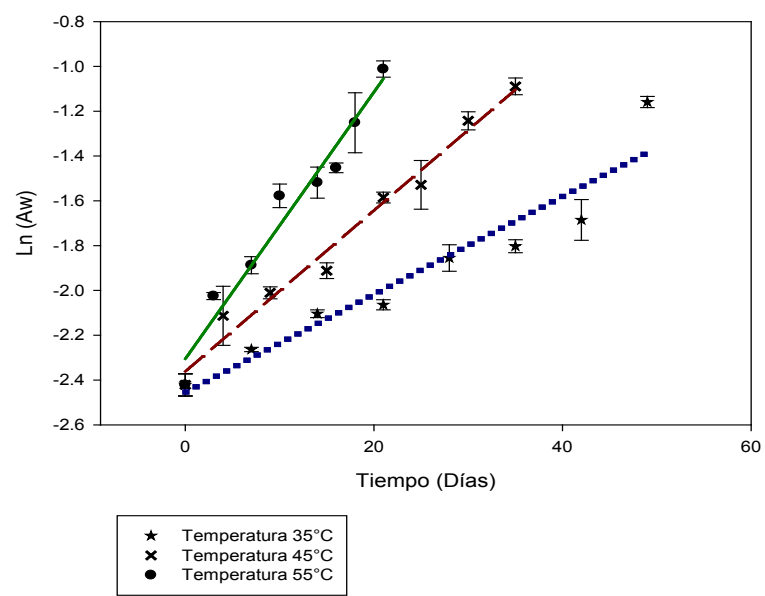

Figura 4. Relación entre $\operatorname{Ln}\left(\mathrm{A}_{\mathrm{w}}\right)$ en función del tiempo de almacenamiento (días) de las galletas a 35,45 y $55^{\circ} \mathrm{C}$.

Una pendiente excesiva $(\mathrm{Ea} / \mathrm{R})$ significa que la reacción es más dependiente de la temperatura; es decir, a medida que la temperatura se incrementa, la reacción aumenta a una velocidad mayor (Valentas et al., 1997). Además, el contenido de humedad y la actividad de agua pueden influenciar los parámetros cinéticos (Ea, Ko), las concentraciones de los reactantes y en algunos casos el orden de reacción aparente (Chica y Osorio, 2003). El parámetro dependiente de la $\mathrm{A}_{\mathrm{w}}$ es la temperatura; asimismo, a medida que aumenta, lo hace la $\mathrm{A}_{\mathrm{w}}$ porque aumenta la presión de vapor (Ordoñez, 1998); lo cual incrementa la facilidad de migración del vapor de agua a través del empaque a la galleta.

El tiempo de vida de la galleta de soda considerando como característica crítica a la actividad de agua fue de 179 días (seis meses), superior al obtenido considerando como parámetro crítico a la humedad (b. s.), pero inferior a lo reportado a DIGESA, 2015 (siete meses). La diferencia entre el valor experimental y el teórico se debe principalmente a que la unidad experimental evaluada fue un paquete de galletas, a diferencia de la presentación de las galleta de soda que tenían un empaque secundario que actuaba como barrera al vapor de agua, alargando su tiempo de vida.

Tabla 5. Determinación de la Energía de activación, Ko, $\mathrm{K}_{20} \mathrm{y}$ el tiempo de vida respecto a la actividad de agua de las galletas de soda.

\begin{tabular}{cccccc}
\hline -Ea (KJ/mol) & Ko & $\begin{array}{c}\mathrm{K}_{20} \\
(\text { día-1) }\end{array}$ & $\mathrm{A}_{\text {wc }}$ & $\mathrm{t}_{20}$ (días) $\mathrm{t}_{20}$ (meses) \\
\hline 42,1661 & 307565,809 & 0,009343 & 0,474 & 179 & 6
\end{tabular}

Awc, actividad de agua critica; Ea, energía de activación; $\mathrm{k}_{20}$, constante de cinética de reacción a $20^{\circ} \mathrm{C} ; \mathrm{K}_{0}$, Factor pre-exponencial; $\mathrm{t}_{20}$, tiempo de vida a $20^{\circ} \mathrm{C}$

\section{Determinación del tiempo de vida a partir de la dureza instrumental}

En la Figura 5 se presenta la variación de dureza instrumental en gramos fuerza (gf) de las galletas de soda respecto al tiempo de almacenamiento a 35,45 y $55^{\circ} \mathrm{C}$. Se observa que a $55^{\circ} \mathrm{C}$ la dureza instrumental disminuyo en mayor grado a comparación de las temperaturas de 35 y $45^{\circ} \mathrm{C}$.

2D Graph 19

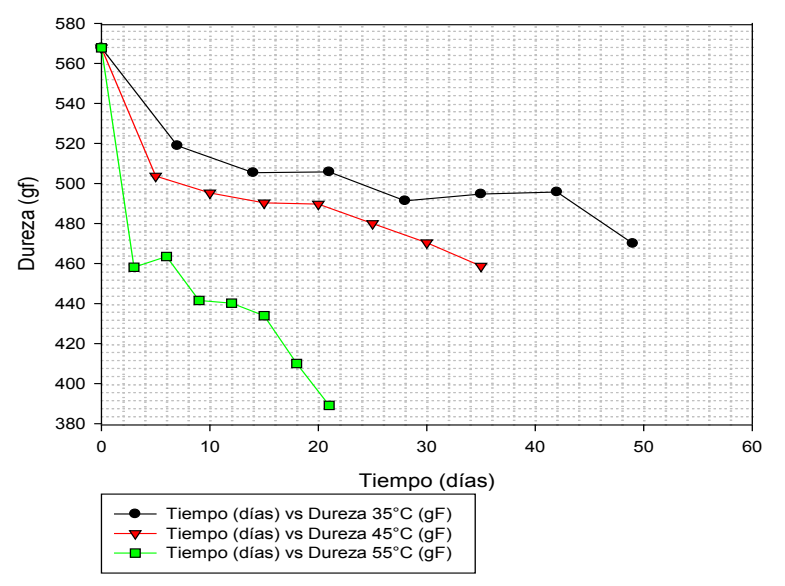

Figura 5. Tendencia de los valores de dureza instrumental respecto al tiempo de almacenamiento de las galletas a 35 , 45 y $55^{\circ} \mathrm{C}$.

Para determinar parámetros texturales en forma instrumental se utilizó la prueba de quiebre de tres puntos, que corresponde a una prueba de flexión en que se evalúa la fuerza máxima necesaria para producir un quiebre total de la estructura del producto (Gaines, 1994 citado por Gewerc y Muñoz, 2006), La textura es el parámetro de calidad de mayor importancia para el consumidor de productos horneados y es utilizada para evaluar la dureza y fracturabilidad de galletas y barras de chocolate, entre otros productos (Castro et al., 2003, citados por Gewerc y Muñoz, 2006). La prueba de quiebre de tres puntos, se caracterizó por ser un método de alta variabilidad de resultados y poca precisión. No se observa una tendencia definida entre la dureza y las respectivas temperaturas de almacenamiento 
(Gewerc y Muñoz, 2006). Lo cual quedó evidenciado en esta investigación y podemos asumir que esto se debe a que si bien las galletas pueden ser geométricamente similares, no lo son en cuanto a los espacios de aire internos, así como que galletas provenientes del mismo lote no tenían el mismo color de tostado, esto podría asumirse a los tiempos de cocción.

Se observa que los valores obtenidos se ajustan mejor a un orden de reacción uno para lo cual se determinaron los valores de coeficiente de determinación $\left(\mathrm{r}^{2}\right)$ y la constante de cinética de reacción $(\mathrm{k})$ para las tres rectas.

Tabla 6. Valores de constante de cinética $(\mathrm{k})$ de reacción para cada temperatura $\left(35,45\right.$ y $\left.55^{\circ} \mathrm{C}\right)$

\begin{tabular}{cccc}
\hline $\mathrm{T}\left({ }^{\circ} \mathrm{C}\right)$ & $\mathrm{r}^{2}$ & $\begin{array}{c}\text { Pendiente } \\
\left(\text { día }^{-1}\right)\end{array}$ & Intercepto \\
\hline 35 & 0,7614 & $-0,0028$ & 6,2941 \\
45 & 0,7756 & $-0,0046$ & 6,2821 \\
55 & 0,7908 & $-0,0135$ & 6,2465 \\
\hline
\end{tabular}

Los valores de coeficiente de determinación $\left(\mathrm{r}^{2}\right)$, constante de cinética de reacción que viene a ser representada por la pendiente de la recta $(\mathrm{k})$ e intercepto de cada temperatura (Tabla 6) se obtuvieron al modelar los datos a una ecuación linealizada de orden uno, donde la abscisa fue el tiempo en días y la ordenada el logaritmo neperiano de la dureza instrumental (gf).

En la Figura 6 se muestra la regresión lineal usando el logaritmo neperiano de la dureza instrumental en gramos fuerza respecto al tiempo de almacenamiento a las tres temperaturas en estudio y una humedad relativa de $80 \%$.

2D Graph 20

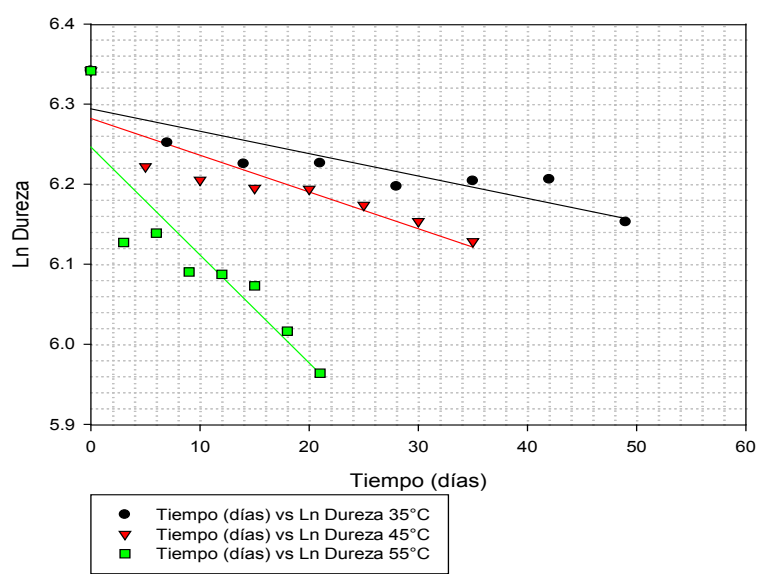

Figura 6. Relación entre Ln(Dureza) en función del tiempo de almacenamiento (días) de las galletas a 35,45 y $55^{\circ} \mathrm{C}$.

En la Tabla 7 se encuentran los valores calculados de energía de activación usando la pendiente de la ecuación por la constante universal de los gases $(0,008314 \mathrm{KJ} / \mathrm{mol}$ $\mathrm{K})$ que proviene de la recta de logaritmo neperiano de las constantes de cinética de reacción y la inversa de la temperatura absoluta correspondiente, mediante Arrhenius se calculó la constante de cinética de reacción para una temperatura de $20{ }^{\circ} \mathrm{C}$ y finalmente usando la ecuación de reacción linealizada de orden uno se estimó el tiempo de vida útil en días y meses a dicha temperatura.

Tabla 7. Determinación de la Energía de activación, Ko, $\mathrm{K}_{20} \mathrm{y}$ el tiempo de vida respecto a la dureza instrumental de las galletas de soda.

\begin{tabular}{cccccc}
\hline Ea $(\mathrm{KJ} / \mathrm{mol})$ & $\mathrm{Ko}$ & $\mathrm{K}_{20}\left(\right.$ día $\left.^{-1}\right)$ & $\mathrm{D}_{\mathrm{c}}(\mathrm{gf})$ & $\mathrm{t}_{20}$ (días) $\mathrm{t}_{20}$ (meses) \\
\hline 65,8195 & 567,7556 & 0,000687 & 471,2314 & 271 & 9 \\
\hline $\mathrm{D}_{\mathrm{c}}$, dureza critica; Ea, energía de activación; $\mathrm{k}_{20}$, constante de cinética de
\end{tabular}
reacción a $20{ }^{\circ} \mathrm{C} ; \mathrm{K}_{0}$, Factor pre-exponencial; $\mathrm{t}_{20}$, tiempo de vida a $20{ }^{\circ} \mathrm{C}$

El tiempo de vida de la galleta de soda para el atributo de dureza instrumental es de 271 días, calculado con la con la ecuación de cinética de reacción de orden uno y con una constante de cinética de reacción para $20^{\circ} \mathrm{C}$,

Kilcast y Subramaniam (2000) citados por Basilio (2015), consideran que la determinación de la vida en anaquel se puede realizar mediante mediciones físicas, siendo la más común la del cambio de textura de un producto. Estos cambios pueden ser el resultado de reacciones químicas que ocurren dentro del producto, como aquellos causados por la interacción entre los ingredientes o por influencia medio ambiental, como la migración de la humedad a través del empaque.

La pérdida de crocantes en los alimentos se produce por absorción de humedad. El agua afecta la textura por ablandamiento y plastificación (Labuza y Katz, 1981 citados por Basilio, 2015), esto tiene relación en la presente investigación ya que las gráficas de ganancia de humedad son proporcionales a la perdida de textura.

\section{Recuento de mohos}

En el Tabla 8 se encuentran los valores del recuento de mohos realizado a las muestras de galleta al inicio del experimento y al final del almacenamiento para cada temperatura, por duplicado, para las temperaturas de 35 , 45 y $55^{\circ} \mathrm{C}$, y ambiente.

Tabla 8. Recuento de mohos en muestras de galleta para las temperaturas de almacenamiento de 35,45 y $55^{\circ} \mathrm{C}$, al inicio y al final del almacenamiento.

\begin{tabular}{cccccc}
\hline \multirow{2}{*}{$\begin{array}{c}\text { Temperatura } \\
\left({ }^{\circ} \mathrm{C}\right)\end{array}$} & Repetición & \multicolumn{2}{c}{$\begin{array}{c}\text { Recuento inicial } \\
(\mathrm{ufc} / \mathrm{ml})\end{array}$} & \multicolumn{2}{c}{$\begin{array}{c}\text { Recuento final } \\
\text { (ufc/ml) }\end{array}$} \\
\cline { 3 - 6 } & & $10^{-1}$ & $10^{-4}$ & $10^{-1}$ & $10^{-4}$ \\
\hline \multirow{2}{*}{35} & $\mathrm{R} 1$ & $<10$ & 1 & $<10$ & 10 \\
& $\mathrm{R} 2$ & $<10$ & 1 & $<10$ & 9 \\
\multirow{2}{*}{45} & $\mathrm{R} 1$ & $<10$ & 1 & $<10$ & 3 \\
& $\mathrm{R} 2$ & $<10$ & 1 & $<10$ & 4 \\
55 & $\mathrm{R} 1$ & $<10$ & 1 & 1 & 3 \\
& $\mathrm{R} 2$ & $<10$ & 1 & 1 & 2 \\
\hline
\end{tabular}


En la Tabla 8, se observa que a medida que se incrementa la temperatura, se va reduciendo el conteo de mohos en las muestras de galleta soda, esto puede deberse a que la temperatura de incubación es un factor primordial para el crecimiento y metabolismo de las células. A medida que la temperatura aumenta las reacciones bioquímicas al interior de la célula se aceleran, por lo cual hay un mayor número de UFC de mohos en el recuento final (temperaturas de 35,45 y $55^{\circ} \mathrm{C}$ ) que en el recuento inicial (temperatura de $\left.20^{\circ} \mathrm{C}\right)$.

Sin embargo, para valores de 45 y $55{ }^{\circ} \mathrm{C}$ se observa que disminuye el recuento de UFC de mohos, esto puede deberse a que las proteínas y los ácidos nucleicos, principalmente, pueden ser desnaturalizados lo cual causa una inhibición en el crecimiento microbiano de los mohos (Sevastianos y Gonzales, 1999).

Todos los valores encontrados al final del almacenamiento, no superan el valor límite máximo permitido sugerido por la Norma Técnica Peruana 206.001 (2016) con respecto al recuento de mohos de galletas $\left(10^{2}\right.$ $\mathrm{UFC} / \mathrm{g})$.

\section{Conclusiones}

Se logró determinar el tiempo de vida útil de las galletas saladas mediante pruebas aceleradas, considerando como parámetros críticos a la humedad, actividad de agua y dureza instrumental, siendo la humedad la que obtuvo el menor tiempo de vida útil (123 días a $20^{\circ} \mathrm{C}$ ), por ende este valor es el tiempo de vida de las galletas saladas en estudio. Además, el recuento de mohos en las muestras de galletas saladas fue menor a lo sugerido por la Norma Técnica Peruana 206.001 durante todo el tiempo que duró el estudio.

\section{Literatura citada}

Basilio, J.E. 2015. Predicción de la vida útil de chifles de plátanos (Musa paradisiaca) mediante modelos matemáticos (Tesis Magister Scientiae). Universidad Nacional Agraria la Molina. Lima, Perú.

Bustamante-Oyague, B. 2015. Estudio de la vida útil de galleta salada mediante la ecuación de Arrhenius (Tesis pregrado). Universidad Nacional del Callao. Lima, Perú.

Carrillo, M.L. y Reyes, A. 2013. Vida útil de los alimentos. Revista Iberoamericana de las Ciencias Biológicas y Agropecuarias: CIBA 2(3): 3.

Cauvain, S. P. and Young, L. S. 2009. Bakery food manufacture and quality: water control and effects. John Wiley \& Sons.

Chica, B. y Osorio, S. 2003. Determinación de la vida de anaquel del chocolate de mesa sin azúcar en una película de polipropileno biorientado (Trabajo de grado para optar al título de Ingeniero Químico). Universidad Nacional de Colombia. Caldas, Colombia.
CPI (Compañía peruana de estudios de mercado y opinión pública). (20129. Mercado de Productos de Consumo y uso personal. no.5. Disponible en: http://www.cpi.pe/ images/upload/paginaweb/archivo/26/mr201205-01. pdf.

Derossi, A.; Mastrandrea, L.; Amodio, ML.; De Chiara MLV. and Colelli, G. (2016). Application of multivariate accelerated test for the shelf life estimation of fresh-cut lettuce. Journal of Food Engineering 169: 122-130.

DIGESA. 2015. Consulta de registros sanitarios. Galleta de Soda. Disponible en: http://www.digesa.minsa.gob. pe/Expedientes/Consulta_Registro_Sanitario.aspx

Espinoza, C. 1993. Determinación de Actividad de agua en Leche y en Jugo de Maracuyá (Passiflora edulis variedad flavicarpa degener) a diferentes concentraciones mediante el método de la depresión del punto de congelación (Tesis pregrado). Universidad Nacional Agraria La Molina, Lima, Perú.

Garcia,Z. 2016. Efecto de la sustitución parcial de harina de trigo (Triticum aestivum) por harina de tarwi (Lupinus mutabilis) sobre las características fisicoquímicas y aceptabilidad general de galletas tipos soda (Tesis pregrado). Universidad Privada Antenor Orrego, Liam, Perú. Disponible en: http://repositorio.upao. edu.pe/bitstream/upaorep/2468/1/RE IND.ALIM FLOR.GARCIA EFECTO.DE.LA.SUSTITUCION. PARCIAL.DE.HARINA.DE.TRIGO_DATOS.PDF

Gewerc, V.A y Muñoz, G.A. 2006. Desarrollo de una galleta tipo snack en base a algas comestibles con enfoque al mercado asiático (memoria para optar al título de ingeniero en alimentos), Universidad de Chile, Facultad de Ciencias Químicas y Farmacéuticas, Departamento de ciencia de los alimentos y tecnología Química.

Granito, M.; Valero, Y.; Zambrano, R. y Guerra, M. 2006. Desarrollo y caracterización de una galleta extendida con caraotas blancas. Agro.Trop.56 (4):539-546. 2006.

Hao, F; Lu, L. and Wang, J. (2016). Finite element simulation of shelf life prediction of moisture-sensitive crackers in permeable packaging under different storage conditions. Journal of Food Processing and Preservation 40: 37-47.

Hough, G. 2010. Sensory Shelf Life Estimation of Food Products. CRC Press, Boca Raton, FL. 264 pp.

Hough, G.; Langohr, K.; Gómez, G. and Curia, A. 2003. Survival analysis applied to sensory shelf life of foods. Journal of Food Science 68: 359- 362.

INACAL. (2016). NTP 206.001. Panadería, Pastelería y Galletería. Galletas. Requisitos. 7p.

Labuza, T. 1979. A theoretical comparison of losses in foods under fluctuating temperatura sequences. $J$. Food Sci. 44:1162.

Manley, D. 2000. Tecnología de la industria galletera. Galletas, crackers y otros horneados. Segunda Edición. Editorial Acribia. Zaragoza, España. 
NTP 206.001. 2016. Panadería, pastelería y galletería. Galletas. Requisito.

NTP 206.011. 1981. Bizcochos, Galletas, Pastas Y Fideos. Determinación de humedad.

Ordoñez J.A. 1998. Tecnología de los alimentos. Madrid, ES. Editorial Síntesis S.A. España. Editorial Síntesis, Vol I, $365 \mathrm{p}$.

Qi, L.; Xu, M.; Fu, Z.; Mira, T. and Zhanga, X. 2014. C2SLDS: A WSNbased perishable food shelf-life prediction and LSFO strategy decision support system in cold chain logistics. Food Control 38: 19-29.

Rao, Q.; Rocca-Smith, JR.; Schoenfussa, TC.; Labuza, TP.; (2012). Accelerated shelf-life testing of quality loss for a commercial hydrolysed hen egg white powder. Food Chem. 135: 464-472.

Rodríguez, V. (2004. Estimación de la vida útil de la harina de pejibae, obtenida por deshidratación (Tesis de Licenciatura en Tecnología de Alimentos). Universidad de Costa Rica, San José, Costa Rica.

Sánchez-González, J. A y Pérez, J.A. 2016. Vida útil sensorial del queso mantecoso por pruebas aceleradas. Scientia Agropecuaria 7 (3): 215 - 222.

Sevastianos, J. y Gonzales, V. 1999. Influencia de la temperatura sobre, el crecimiento de hongos termófilos y termotolerantes. VIII Congreso Nacional de Biolecnologia y Bioingenieria.

Sirpatrawan, U. 2009. Shelf life simulation of packaged rice crackers. J. Food Qual. 32, $224-239$ p.

Subramaniam, P y Wareing, P. 2016. The Stability and Shelf Life of Food. Segunda edición. Woodhead Publishing. 612 p.

Udomkun, P.; Nagle, M.; Argyropoulos, D.; Mahayothee, B.; Latif, S. and Müller, J. 2016. Compositional and functional dynamics of dried papaya as affected by storage time and packaging material. Food Chem. 196: 712-719.

Valentas K.J., Rostein E., Singh R.P. 1997. Handbook of Food Engineering Practice. CRC Press. Boca Raton New York. 